\title{
NUEVOS SISTEMAS DE RENDICIÓN DE CUENTAS DE LA INFORMACIÓN PERIODÍSTICA. EXPLORACIÓN DEL ESCENARIO ONLINE ESPAÑOL
}

\author{
New media accountability systems. Exploration of the \\ Spanish online environment
}

Marcel Mauri-Ríos y Xavier Ramon-Vegas
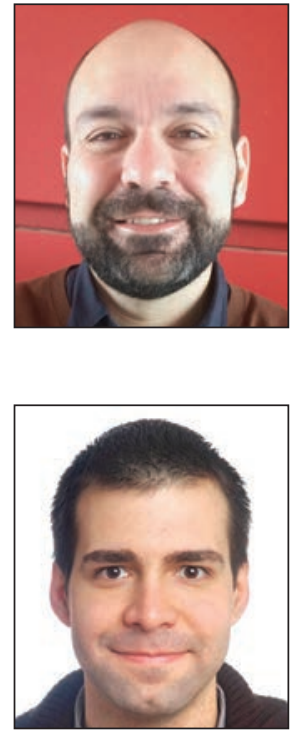

Marcel Mauri-Ríos es doctor en periodismo por la Universitat Pompeu Fabra (UPF). Sus principales líneas de investigación son la historia del periodismo y la deontología periodística, el periodismo de calidad y la rendición de cuentas. Es profesor e investigador del Departamento de Comunicación de la UPF y es consultor de ética y derecho de la información en la Universitat Oberta de Catalunya (UOC). Es miembro del Grup de Recerca en Periodisme y participa en el proyecto competitivo I+D+i Transparencia y rendimiento de cuentas de la información periodística (Ministerio de Economía y Competitividad, Ref.: CSO2012-39138).

http://orcid.org/0000-0003-2615-8343

marcel.mauri@upf.edu

Xavier Ramon-Vegas es profesor e investigador del Departamento de Comunicación de la Universitat Pompeu Fabra (UPF). Doctorando en comunicación, es Master en estudios avanzados en comunicación social y licenciado en comunicación audiovisual y periodismo por la UPF. Sus principales líneas de investigación son la ética y la calidad informativa, el periodismo deportivo y los estudios olímpicos. Es miembro del Grup de Recerca en Periodisme y participa en el proyecto competitivo I+D+i Transparencia y rendimiento de cuentas de la información periodística (Ministerio de Economía y Competitividad, Ref.: CSO2012-39138).

http://orcid.org/0000-0002-4478-5626

xavier.ramon@upf.edu

Universitat Pompeu Fabra, Departament de Comunicació Roc Boronat, 138. 08018 Barcelona, España

\section{Resumen}

En un contexto en profunda transformación, los sistemas de rendición de cuentas de la información periodística han experimentado una clara evolución al aprovechar las posibilidades de internet y la web 2.0. Mientras que los instrumentos tradicionales tienen un impacto limitado en la práctica profesional y un uso escaso por parte de los ciudadanos, el entorno digital favorece la aparición de nuevas formas de transparencia, autorregulación y supervisión de la calidad informativa, en las que la participación de los usuarios juega un papel decisivo. Se identifican, describen y categorizan los nuevos sistemas de rendición de cuentas online surgidos en el panorama mediático español, examinando tanto los que operan dentro de los propios medios digitales como fuera de ellos. El análisis de los casos detectados ha posibilitado elaborar una primera clasificación de los sistemas innovadores de rendición de cuentas existentes en España.

\section{Palabras clave}

Rendición de cuentas; Periodismo; Calidad informativa; Transparencia; Autorregulación; Participación.

\footnotetext{
Abstract

In a profoundly changing context, media accountability systems have clearly undergone a process of evolution by taking advantage of the internet and web 2.0 possibilities. While traditional instruments have a limited impact on professional practice and are scarcely used by citizens, the digital environment allows new ways of self-regulation, transparency, and monitoring of news quality, in which users' participation plays a decisive role. The article identifies, describes and categorizes the new online accountability systems that have emerged in the Spanish media landscape, examining those that operate both within and outside digital media. The analysis of the identified systems has allowed the elaboration of a first classification of the innovative accountability systems existing in Spain.
} 


\section{Keywords}

Accountability; Journalism; News quality; Transparency; Self-regulation; Participation.

Mauri-Ríos, Marcel; Ramon-Vegas, Xavier (2015). “Nuevos sistemas de rendición de cuentas de la información periodística. Exploración del escenario online español". El profesional de la información, v. 24, n. 4, pp. 380-389.

http://dx.doi.org/10.3145/epi.2015.jul.04

\section{Introducción}

Existe una coincidencia generalizada acerca de la importancia de los medios de comunicación para la salud de cualquier democracia. En base a esta convicción, las legislaciones nacionales e internacionales reconocen ampliamente el derecho a la información, cuya titularidad recae en los ciudadanos. Por ese motivo es esencial conocer los sistemas que utilizan los medios de comunicación para rendir cuentas sobre su acción. Estos sistemas de rendición de cuentas o media accountability systems (Bertrand, 2000) son indicadores clave del pluralismo y la transparencia de los medios en un estado democrático. Esto es así en la medida que su función esencial es supervisar, controlar, criticar y examinar la evolución y calidad de la información periodística, y más en un contexto de crisis del sector y de concentración mediática (Eberwein, 2010).

La rendición de cuentas es un concepto que hace referencia a la voluntad de los medios de responder ante la sociedad por la actividad que llevan a cabo. Rendir cuentas "está habitualmente vinculado a aceptar ciertas responsabilidades, tareas u objetivos" (Christians et al., 2009, p. 132) y se traduce en la voluntad periodística de ejercer la autorregulación profesional, la transparencia informativa y fomentar la participación del público. En la medida que un medio fomenta estos tres valores profesionales se puede considerar que ejerce la rendición de cuentas para con el público.

En un contexto periodístico en profunda transformación, los sistemas de rendición de cuentas han experimentado una clara evolución en los últimos años al aprovechar las posibilidades de internet y la web 2.0 (Fengler et al., 2014). Los instrumentos de rendición de cuentas tradicionales (defensores del lector offline, códigos éticos, cartas al director, entre otros), tienen un impacto limitado en la práctica profesional y un uso escaso por parte de los ciudadanos (Alsius; Salgado, 2010). Pero el entorno digital fomenta nuevas formas de transparencia y control de la calidad informativa, a la vez que posibilita a los ciudadanos participar y comentar la calidad de los medios (Pastor; Martínez-Martínez, 2013).

Hasta el momento la mayoría de las investigaciones sobre este tema se ha centrado en los formatos clásicos, como:

- códigos deontológicos (Barroso, 1984; Nordenstreng; Hannikainen, 1984);

- códigos internos y libros de estilo (Aznar, 1999; Alsius, 1999; García-Avilés, 1996; Pérez-Fuentes, 2004);

- defensores del lector (Evers; Groenhart; Van-Groesen, 2010; Maciá-Barber, 2006; Starck, 2010).

Sin embargo, como respuesta al desarrollo incipiente de sistemas innovadores, en Europa y Estados Unidos han aparecido recientemente trabajos sobre los nuevos medios de rendición de cuentas en internet (Domingo; Heinonen, 2008; Eberwein, 2010; Fengler et al., 2014). En cambio, en España son muy escasas las aportaciones sobre esta cuestión más allá de las primeras aproximaciones de HerreraDamas (2013) o Alsius et al. (2011).

En este artículo se presentan los resultados de una investigación que, partiendo de la voluntad de conocer los mecanismos innovadores surgidos con internet y la web 2.0, identifica, describe y categoriza los sistemas de rendición de cuentas existentes dentro y fuera de los medios.

Rendir cuentas en entorno digital significa ejercer la autorregulación profesional, la transparencia informativa y fomentar la participación de los usuarios

\section{Metodología}

El trabajo utiliza varios métodos. Dado que el fin de la investigación era conocer la existencia de nuevos sistemas de rendición de cuentas en internet y la web 2.0, se partía de un desconocimiento de la muestra a inspeccionar. Por ese motivo se utilizó una metodología basada en el sistema de muestreo no probabilístico conocido como bola de nieve o snowball sampling (Goodman, 1961). Esta técnica, indicada para investigaciones de poblaciones muy dispersas pero en contacto entre sí, se fundamenta en ir identificando sujetos -o experiencias- que se van incorporando progresivamente a la muestra.

Se partió de los datos aportados por un panel de expertos (investigadores españoles especialistas en autorregulación periodística, representantes de entes de autorregulación y profesionales) realizado en julio de 2013. Estos agentes proporcionaron indicaciones sobre personas, organismos o instrumentos innovadores que conocían. Se añadieron como guía de búsqueda las experiencias detectadas por Fengler et al. (2014), que realizaron un primer mapeo de sistemas de rendición de cuentas online en países europeos en el marco del proyecto MediaAct (Comisión Europea, SSH-2009-5.1.1).

El trabajo de campo posterior consistió en rastrear por internet, a partir de las primeras experiencias aportadas por los expertos, y tirar del hilo hasta obtener los sistemas de rendición de cuentas más novedosos. Se delimitó un marco temporal de rastreo amplio, de doce meses (febrero de 2014 - enero de 2015), necesario en un tipo de investigación donde el universo es infinito y se desconoce la amplitud de la muestra. 
Una vez identificados los sistemas relevantes, se procedió a su análisis de contenido cualitativo, también denominado análisis del contenido etnográfico (Altheide, 1996; Bryman, 2012). En su aplicación, se contemplaron las pautas señaladas por Wimmer y Dominick (2000, pp. 141-144).

Tomando como base el documento Best practice guidebook de MediaAct (Bichler et al., 2012), se construyó un esquema de codificación con las categorías:

- producción interna o externa a los medios;

- descripción de las especificaciones y uso del instrumento;

- formas de participación del público;

- y valoración del mecanismo para la rendición de cuentas.

En última instancia, se procedió a clasificar y categorizar las experiencias detectadas.

\section{Resultados}

Se detectaron los sistemas de rendición de cuentas online que se describen a continuación, agrupados en dos categorías:

- producidos en empresas o grupos mediáticos;

- implementados de forma externa a estas empresas o grupos.

\subsection{Sistemas producidos en empresas o grupos me- diáticos}

Son:

- blogs de redacciones de medios;

- blogs de periodistas incluidos en la web del medio;

- defensores del lector online;

- chats y encuentros digitales con los lectores;

- contribución de los usuarios en la creación de contenidos;

- botones de notificación de errores;

- redes sociales y comentarios;

- instrumentos de transparencia corporativa.

A continuación se detallan, aportando ejemplos relevantes del panorama español.

\section{Blogs de redacciones de medios}

A nivel internacional destacan blogs de redacciones que, desde la óptica de la rendición de cuentas, fomentan la transparencia de la tarea periodística y abren un proceso de reflexión pública sobre ésta. Entre las experiencias más significativas, cabe remarcar:

- Editor's blog de la BBC http://www.bbc.com/news/blogs/the_editors

- Espacio del periódico Spokesman-Review http://www.spokesman.com/blogs/editors-notes

- Blogs de los medios alemanes Zeit http://blog.zeit.de/zeitansage
- INICIAR SESION $\rightarrow$ REGISTRO Buscar Buscar.

EL BLOG DE LA REDACCIÓN eldiario.es

SOBRE ESTE BLOG

Y Seguir a Geldiarioes

ff Mo gusta 210392

En este blog te contaremos desde dentro todas las novedades del proyecto de eldiario.es. Puedes escribirnos en los comentarios o en contacto@eldiario.es.

BUSCADOR
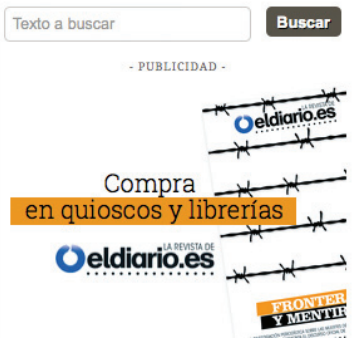

- Tagesschau

http://blog.tagesschau.de

- $T a z$

http://blogs.taz.de/hausblog

- Blog Interna del rotativo suizo Südostschweiz http://www.suedostschweiz.ch/community/blogs/interna

En España sobresale el blog de la redacción de Eldiario.es, creado en octubre de 2010. En él se explican desde dentro las novedades del proyecto editorial del medio. Asimismo se relatan las interioridades de coberturas especiales, se retransmiten debates en directo y se hace referencia a la participación de los periodistas en actos y presentaciones. Desde su creación se han publicado 132 entradas, con un promedio de actualización de 2,54 entradas por mes. Sin embargo, se percibe una falta de delimitación más clara en-

Tabla 1. Nuevos sistemas de rendición de cuentas en el escenario español

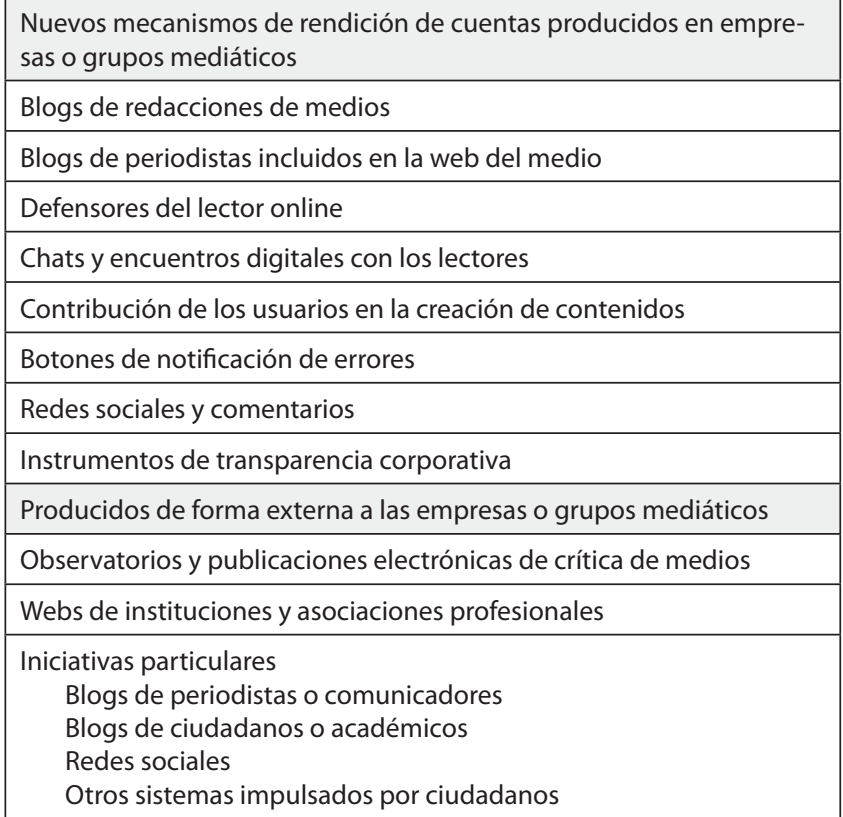


tre las entradas dedicadas a los procesos informativos y las centradas en la promoción cruzada de productos como libros, revistas o ediciones regionales.

http://www.eldiario.es/redaccion

\section{Blogs de periodistas incluidos en la web del medio}

Determinados medios también han apostado por alojar blogs de periodistas que monitorizan la calidad de los contenidos del sector, como la de Eric Wemple en The Washington post.

http://www.washingtonpost.com/ blogs/erik-wemple

Este tipo de blogs cuenta con ejemplos relevantes en España:

\section{3grados \\ http://233grados.lainformacion.com}

Es una plataforma puesta en marcha por periodistas de Lainformacion.com, que no sólo examina la actualidad y las tendencias del sector sino que también reflexiona críticamente sobre la tarea de los medios. Se aborda un amplio abanico de cuestiones éticas, como la manipulación de las imágenes, la credibilidad de las fuentes, la invasión de la privacidad o la violencia contra los periodistas. Desde mayo de 2008 se han publicado 8.445 entradas.

\section{Crítica periodística \\ http://www.eldiario.es/catalunya/criticaperiodistica}

Impulsada por Eldiario.es y conducida por el catedrático en periodismo Jaume Guillamet. El autor reflexiona sobre cuestiones éticas de primer orden, aportando en sus textos una visión que vincula el contexto actual con momentos clave de la historia del periodismo.

\section{Defensores del lector online}

A nivel internacional hay referentes como:
- Margaret Sullivan, The New York times http://publiceditor.blogs.nytimes.com

- Edward Schumacher-Matos, NPR http://www.npr.org/blogs/ombudsman

- Chris Elliott, The guardian http://www.theguardian.com/commentisfree/series/ open-door

- Lutz Büge, Frankfurter Rundschau http://www.frblog.de

En España, dos blogs destacan por la profundidad de sus textos y la regularidad de actualización:

- José Cervera, defensor de la comunidad de Eldiario.es http://www.eldiario.es/defensor

- Lola Galán, defensora de El país, http://blogs.elpais.com/defensor-del-lector

Ambos invitan al público a debatir sobre cuestiones éticas y la calidad periodística. En Eldiario.es, los lectores pueden utilizar un formulario para mandar sus inquietudes al defensor, que estudia los mensajes de acuerdo con los principios editoriales y la ética profesional del medio. Posteriormente, se da una respuesta pública en el blog a los mensajes que "se consideren de más peso". En el caso de El país se puede contactar con la defensora mediante carta, correo electrónico o teléfono.

Desde septiembre de 2012 el blog de Eldiario.es ha publicado 62 entradas (2,14 por mes). El blog de El país cuenta con 126 entradas, 15 redactadas por Galán y 111 por el anterior defensor, Tomàs Delclós. Sumando ambas etapas, el promedio mensual de entradas es de 3,60.

Figura 3. Pregúntame, encuentros digitales con los lectores de Eldiario.es 


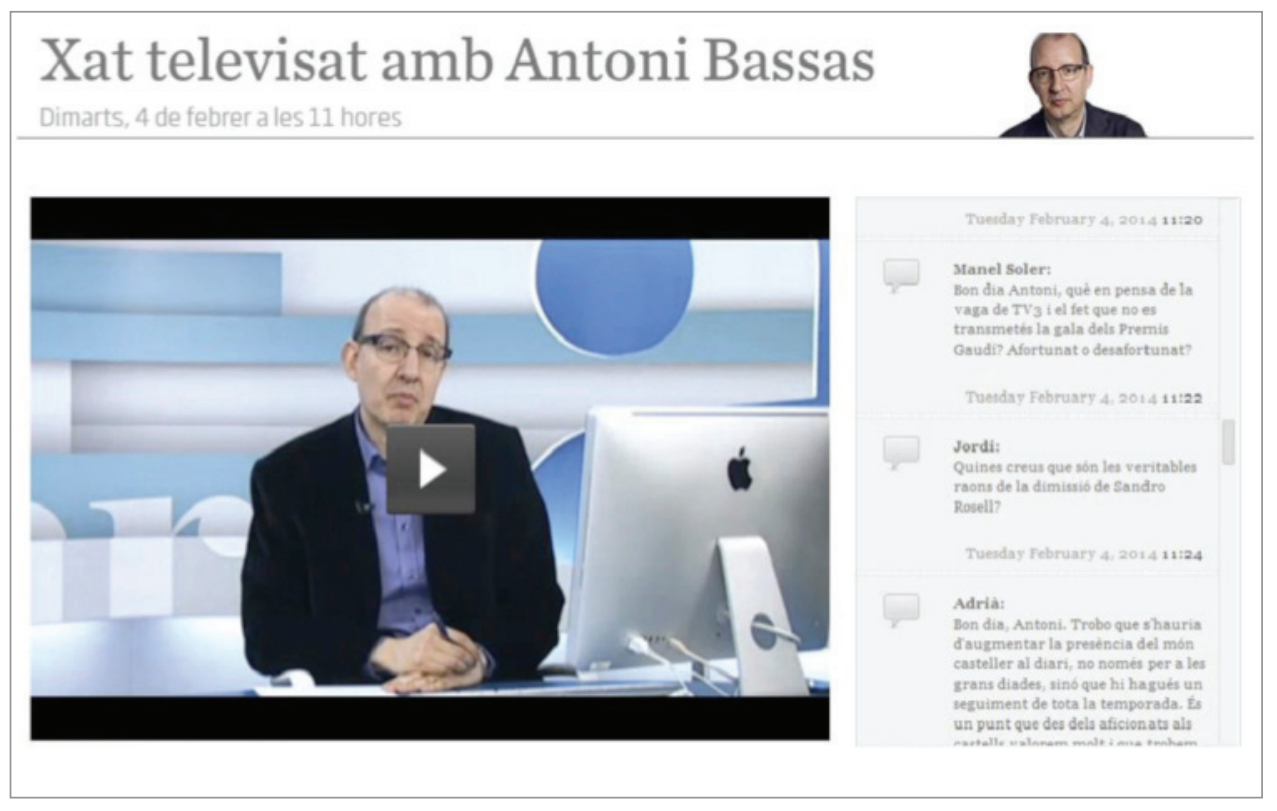

Figura 4. Chat televisado con los lectores del diario Ara

\section{Chats y encuentros digitales con los lectores}

Varios medios han desplegado mecanismos de interacción en tiempo real entre los lectores, el equipo editorial y expertos. Un ejemplo ilustrativo es The Washington post, que en julio de 2014 impulsó los chats Live Q\&A (questions \& anwers). Desde entonces se han conducido cerca de 500 encuentros, consultables íntegramente online.

http://live.washingtonpost.com

En España Eldiario.es lanzó en enero de 2015 los encuentros digitales Pregúntame, en colaboración con el portal Menéa$m e$. En el primero de estos encuentros, los lectores pudieron interactuar con el director del medio, Ignacio Escolar. https://www.meneame.net/m/preguntame

Cabe puntualizar que fuera de la Web, Eldiario.es también organiza encuentros periódicos, donde los socios pueden debatir con los profesionales sobre la línea editorial.

http://www.eldiario.es/hazte_socio

La marea propone a sus socios "mecenas" participar en encuentros offline, incluyendo la asistencia al consejo de redacción.

http://www.lamarea.com/2014/12/15/nace-el-club-deamigos-y-amigas

Mònica Terribas, la anterior editora del diario Ara creó un chat semanal en formato vídeo streaming para todos los suscriptores o usuarios registrados donde atendía las sugerencias y críticas sobre los contenidos, para después transmitirlas a la redacción. Con el actual editor, Antoni Bassas, el chat se ha mantenido, pese a que no cuenta con la regularidad de la anterior etapa.

http://www.ara.cat/media/Xat-Antoni_Bassas-subscriptors-registrats_0_1078092408.html

\section{Contribución de los usuarios en la creación de contenidos}

Tomando como inspiración experiencias como TexasTribune. org (Estados Unidos) y Rue89.com (Francia), VilaWeb.cat cuenta con una comunidad de lectores, +VilaWeb, que pueden participar en el proceso editorial. La redacción manda un correo cada tarde para informarles de los temas en producción y los suscriptores pueden realizar sus aportaciones, sugerir enfoques y ayudar en la búsqueda de fuentes.

http://www.vilaweb.cat/ mesvilaweb

Además, en el editorial del director Vicent Partal se recogen las opiniones que los suscriptores mandan sobre la pieza en cuestión.

http://www.vilaweb.cat/ editorial

Más allá de la fórmula de la comunidad, otros sistemas que fomentan la participación de los usuarios en la creación de contenidos son los canales dedicados a las contribuciones de los lectores. Algunos ejemplos destacados son:

- sección Los socios/as escriben de La marea http://www.lamarea.com/secciones/los-socios-escriben

- Fem crític, el blog de los suscriptores de Elcritic.cat, un medio centrado en el periodismo de investigación. http://www.elcritic.cat/blogs/femcritic

Blogs, chats, redes sociales, botones de corrección u observatorios han generado nuevos espacios de supervisión, reflexión y debate

\section{Botones de notificación de errores}

Las noticias de Lainformación.com, El Huffington post y Libertad digital disponen de un botón que permite a los lectores advertir a la redacción de los errores detectados. En los dos primeros la notificación se efectúa mediante un formulario, mientras que en Libertad digital se realiza por correo electrónico.

http://www.lainformacion.com

http://www.huffingtonpost.es

http://www.libertaddigital.com

\section{Redes sociales y comentarios}

Las redes sociales no son únicamente un canal de difusión: son también un mecanismo de rendición de cuentas. Los ciudadanos pueden monitorizar la calidad de los contenidos y exponer sus quejas mediante Twitter, Facebook y hacer comentarios sobre las noticias. Un ejemplo paradigmático se encuentra en El periódico de Catalunya. El 28 de agosto de 2011 el medio avanzó en Twitter la portada del día siguiente. Esta recogía citas de un informe policial que identificaba grupos de delincuentes extranjeros que operan en 
Barcelona. Los seguidores criticaron el carácter xenófobo de la portada, hecho que llevó al medio a corregir antetítulo y subtítulo ${ }^{1}$. El periódico también dispone de una cuenta específica en Twitter para que el público transmita opiniones, denuncias y recomendaciones.

https://twitter.com/epentretodos

\section{Instrumentos de transparencia cor-} porativa

$\mathrm{Si}$ bien ciertas empresas como $\mathrm{Me}$ diaset o Atresmedia publican online las cuentas de resultados de sus medios, es frecuente que los lectores deban bucear en las webs de dichos grupos comunicativos para localizar estos datos en las memorias anuales. Este hecho limita notablemente el grado de transparencia respecto a los depositarios de la información. En contraste, Eldiario.es detalla de forma clara sus cuentas en el blog del director Ignacio Escolar ${ }^{2}$, pormenorizando las partidas (fuentes de ingresos, gastos, socios, empleados y salarios).

Esta práctica se asemeja a la apuesta realizada por TexasTribune.org, que en su apartado About us facilita de forma rápidamente localizable dicha información.

https://www.texastribune.org/about

\subsection{Sistemas producidos de forma externa a las em- presas o grupos mediáticos}

Son:

- observatorios y publicaciones electrónicas de crítica de medios;

- webs de instituciones y asociaciones profesionales;

- otras iniciativas particulares (blogs de periodistas, blogs de ciudadanos, redes sociales y otros sistemas impulsados por ciudadanos).

\subsubsection{Medios específicos y observatorios de crítica de me- dios}

Hay pocas iniciativas centradas en la monitorización de los contenidos periodísticos en España. Destaca Mèdia.cat, un observatorio de crítica de medios que diariamente señala buenas y malas prácticas a partir del análisis transversal sobre cómo han tratado un hecho informativo concreto. Desde 2011 se elaboran reportajes de investigación en profundidad (compilados en el Anuario Mèdia.cat, que también tiene versión en papel) sobre temas relevantes que han sido silenciados por intereses económicos, políticos o de otro tipo. La plataforma fomenta la participación de los usuarios, expertos y profesionales, invitándoles a redactar informes y a contribuir al análisis mediático.

http://www.media.cat

La existencia de observatorios de medios es más habitual en otros países. Algunas iniciativas:
En Estados Unidos:

- Project Censored http://www.projectcensored.org

- Media Matters for America http://mediamatters.org

- Blog Regret the error del Poynter Institute http://www.poynter.org/tag/regret-the-error

En Francia:

- Sur Le Journalisme http://surlejournalisme.com

- L'Observatoire des médias http://www.observatoiredesmedias.com

En el escenario europeo:

- European Journalism Observatory http://en.ejo.ch

En Latinoamérica:

- Ética Segura, de la Fundación Nuevo Periodismo lberoamericano http://eticasegura.fnpi.org

\subsubsection{Webs de instituciones y asociaciones profesionales}

Algunas instituciones que contribuyen a la autorregulación mediática desde fuera de las empresas, como los consejos de prensa, también han aprovechado internet para ahondar en sus funciones de supervisión ética de los medios. Este es el caso de la web y blog del Consell de la Informació de Catalunya $(\mathrm{ClC})$, organismo independiente vinculado al Col/legi de Periodistes de Catalunya (CPC). El mecanismo más interesante desde la óptica de la rendición de cuentas es un formulario que ayuda a los ciudadanos a presentar escritos de queja al CIC por las infracciones éticas del código 
deontológico cometidas por los medios.

http://fcic.periodistes.org http://fcic.periodistes.org/ presentar-una-queixa-online

La Comisión de Arbitraje, Quejas y Deontología del Periodismo de la Federación de Asociaciones de la Prensa de España (FAPE) también realiza funciones propias de un press council. Desde su web los ciudadanos pueden saber cómo tramitar una queja, si bien no dispone de un formu-

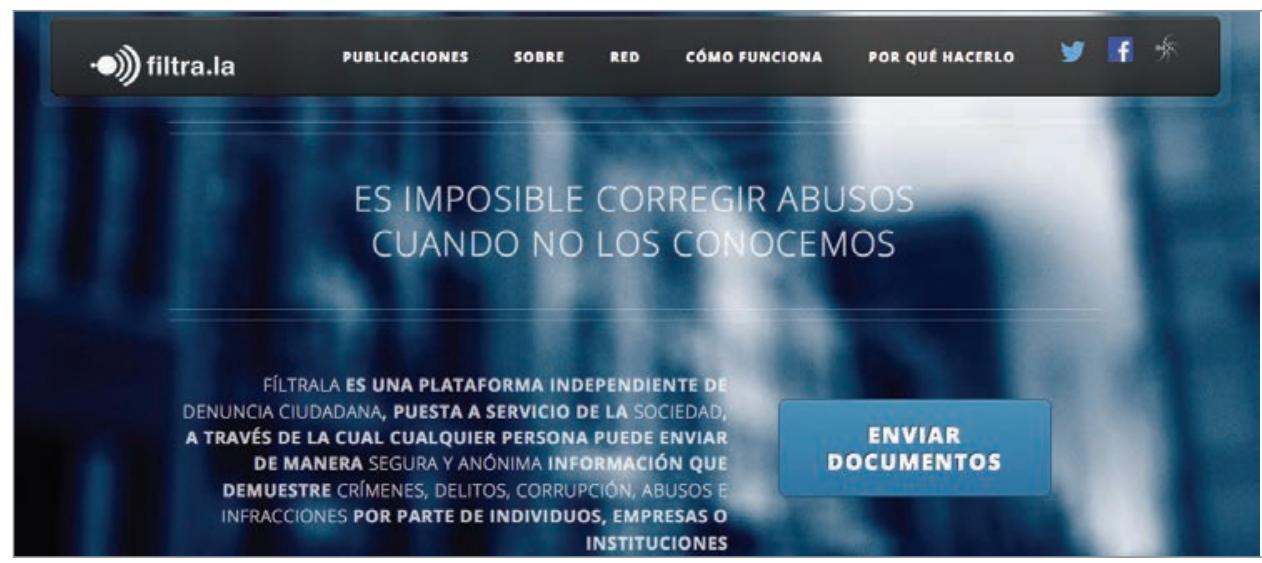

Figura 6. Plataforma de denuncia ciudadana Fíltrala https://filtrala.org

\section{lario online que facilite hacer-}

lo y se deben enviar los documentos por correo postal.

http://www.comisiondequejas.com

En el entorno de las asociaciones de periodistas destaca la web del Grup de Periodistes Ramon Barnils, asociación catalana de profesionales de la información que señala de forma sistemática los déficits informativos de los grandes medios de comunicación.

http://www.grupbarnils.cat

El auge de medios online no ha impedido la aparición de nuevos espacios de relación presencial con el público

\subsubsection{Iniciativas particulares}

\section{Blogs de periodistas o comunicadores}

Algunos periodistas, a título individual y sin vinculación alguna con empresas, mantienen blogs de análisis del comportamiento deontológico de los medios de comunicación. En este trabajo no se pretende presentar un catálogo exhaustivo de blogs sino resaltar algunos que destacan por su relevancia y regularidad de publicación:

- Paper Papers: blog de Toni Piqué y Gonzalo Peltzer, que desde ambos lados del Atlántico aportan reflexiones críticas sobre el ejercicio del periodismo, los contenidos de los medios y su rendición de cuentas.

http://www.paperpapers.net

- María Ramírez y Eduardo Suárez, ex corresponsales de El mundo en los Estados Unidos, son los responsables del blog No hace falta papel, donde observan la innovación de los medios con una perspectiva crítica y atendiendo a la responsabilidad.

http://nohacefaltapapel.com

- También pone el foco en las nuevas tendencias el blog Fcinc de Isaac Salvatierra, director de la edición digital del periódico Ara, si bien su blog no tiene ninguna vinculación con el medio. Desde aquí comenta los cambios del periodismo en la era digital, atendiendo frecuentemente a los fallos y responsabilidades de los medios.

http://www.isaacsalvatierra.cat

\section{Blogs de ciudadanos y académicos}

Con el propósito de monitorizar el panorama mediático desde una perspectiva ética desde fuera de la profesión encontramos blogs elaborados por académicos o por ciudadanos sin vinculación profesional con el periodismo, como:

- La buena prensa: un proyecto del profesor de periodismo de la Universidad de Navarra Miguel Ángel Jimeno que pone el foco en las buenas prácticas profesionales. http://labuenaprensa.blogspot.com.es

- Su antagonista es Mala prensa, un blog de crítica de medios del académico Josu Mezu, doctor en ciencias políticas, que monitoriza un aspecto muy concreto de la calidad de la prensa española: el rigor y la exactitud en los datos y los conceptos.

http://www.malaprensa.com

- Periodismo deportivo de calidad, del profesor de la Universidad de Sevilla José Luis Rojas Torrijos, fomenta la reflexión sobre el lenguaje y la ética en el periodismo deportivo. http://periodismodeportivodecalidad.blogspot.com.es

\section{Redes sociales}

Particularmente Twitter y Facebook, han irrumpido en los últimos años como aplicaciones masivas e influyentes para la rendición de cuentas en la medida que los ciudadanos los usan con la finalidad de criticar directamente a los medios cuando incumplen sus compromisos deontológicos. La necesidad de los medios de tener una ventana abierta en las redes les obliga a dar respuesta a esta crítica constante. En ese sentido las redes sociales son plataformas efectivas y reales de participación ciudadana, de transparencia y de autorregulación periodística.

\section{Otros sistemas impulsados por periodistas o ciudadanos}

Otras iniciativas también contribuyen a la participación de los ciudadanos en el debate sobre la calidad periodística:

- Fixmedia es una web que invita a los ciudadanos a enviar errores (ortográficos y éticos) detectados en las noticias a través de un sencillo formulario. Se inspira en MediaBugs, de Estados Unidos.

http://www.fixmedia.org

http://mediabugs.org

- Fíltrala es una plataforma que habilita a periodistas y 
ciudadanos a enviar información de interés público, que después será examinada a fondo. No aceptan el envío de rumores, sólo de documentación que pueda servir para abrir una investigación periodística.

https://filtrala.org

- Web de la Federación de Asociaciones de Consumidores y Usuarios de los Medios, organización que reúne asociaciones de teleespectadores de España con el fin de promover unos medios de comunicación responsables. Mediante el buzón de quejas, los ciudadanos pueden pedir explicaciones acerca de los contenidos de los medios que juzgan inadecuados.

http://www.icmedianet.org

\section{Discusión y conclusiones}

Nos encontramos en un escenario caracterizado por la adaptación de mecanismos tradicionales a la nueva cultura digital (como los defensores del lector) y por el surgimiento de un amplio abanico de medios novedosos (tabla 2), impulsados por parte de las empresas mediáticas y por agentes externos, como instituciones, asociaciones profesionales, ciudadanos y expertos.

El uso creciente de iniciativas como blogs, chats, redes sociales, botones de corrección u observatorios, ha generado nuevos espacios de supervisión, reflexión y debate sobre la línea editorial y la calidad de los contenidos, en los que la participación del público juega un papel fundamental. A su vez el auge de estos medios online no ha impedido la incipiente aparición de espacios de relación cara a cara con el público (como los consejos de redacción presenciales con los lectores). Todos estos mecanismos online y offline no sólo refuerzan el vínculo medio-usuario sino que otorgan a los lectores el papel de corresponsables del compromiso de llevar a cabo un periodismo riguroso y de calidad.

\section{Las redes sociales no son únicamente un canal de difusión, sino un espacio esen- cial donde monitorizar la calidad perio- dística y exponer quejas}

La investigación señala que también se encuentran limitaciones y aspectos de primer orden a mejorar en el diseño e implementación de estos sistemas de rendición de cuentas.

En primer lugar, existe una falta de constancia y sistematicidad. Por ejemplo, mecanismos como los chats televisados no gozan de una regularidad establecida.

En segundo lugar, se ha apreciado que en ocasiones en el

Tabla 2. Casos detectados en el escenario online español

\begin{tabular}{|c|c|}
\hline Mecanismos & Casos \\
\hline \multicolumn{2}{|c|}{ Producidos en el seno de empresas o grupos mediáticos } \\
\hline Blogs de redacciones de medios & Blog de la redacción de Eldiario.es \\
\hline Blogs de periodistas incluidos en la web del medio & $\begin{array}{l}233 \text { grados } \\
\text { Crítica periodística (Eldiario.es) }\end{array}$ \\
\hline Defensores del lector online & $\begin{array}{l}\text { Defensor de la comunidad de Eldiario.es } \\
\text { Defensora de El país }\end{array}$ \\
\hline Chats y encuentros digitales con los lectores & $\begin{array}{l}\text { Encuentros digitales Pregúntame (Eldiario.es-Menéame) } \\
\text { Chat en formato vídeo con el editor (Ara) }\end{array}$ \\
\hline Contribución de los usuarios en la creación de contenidos & $\begin{array}{l}\text { Comunidad de lectores +VilaWeb (VilaWeb.cat) } \\
\text { Sección Los socios/as escriben (La marea) } \\
\text { Fem crític, blog de los suscriptores de Elcritic.cat }\end{array}$ \\
\hline Botones de notificación de errores & $\begin{array}{l}\text { Lainformación.com } \\
\text { El Huffington post } \\
\text { Libertad digital }\end{array}$ \\
\hline Redes sociales y comentarios & Cuenta EP Entre todos (El periódico) \\
\hline Transparencia corporativa & Cuentas de El diario.es en el blog del director Ignacio Escolar \\
\hline \multicolumn{2}{|c|}{ Producidos de forma externa a las empresas o grupos mediáticos } \\
\hline Observatorios y publicaciones electrónicas de crítica de medios & Mèdia.cat \\
\hline Webs de instituciones y asociaciones profesionales & $\begin{array}{l}\text { Web y blog del Consell de la Informació de Catalunya (CIC) } \\
\text { Web Comisión de Arbitraje, Quejas y Deontología del Periodismo (FAPE) } \\
\text { Web Grup de Periodistes Ramon Barnils }\end{array}$ \\
\hline Blogs de periodistas o comunicadores & $\begin{array}{l}\text { Paper Papers } \\
\text { No hace falta papel } \\
\text { Fcinc }\end{array}$ \\
\hline Blogs de ciudadanos o académicos & $\begin{array}{l}\text { La buena prensa } \\
\text { Mala prensa } \\
\text { Periodismo deportivo de calidad }\end{array}$ \\
\hline Redes sociales & Redes sociales utilizadas por los ciudadanos \\
\hline Otros sistemas impulsados por ciudadanos & $\begin{array}{l}\text { Fixmedia } \\
\text { Fíltrala } \\
\text { Web de la Federación de Asociaciones de Consumidores y Usuarios de los Medios }\end{array}$ \\
\hline
\end{tabular}


uso de ciertos instrumentos se produce una desviación de los propósitos de rendición de cuentas, que puede menoscabar su percepción como tales por parte de los usuarios. Como se ha comentado anteriormente, en el blog de la redacción de Eldiario.es frecuentemente se difuminan las fronteras entre la explicación de los procesos editoriales y la promoción cruzada. $\mathrm{O}$, en los encuentros digitales que organiza este medio con los lectores, ciertas preguntas buscan la opinión personal o el posicionamiento del director sobre una cuestión de actualidad, en lugar de ahondar en la tarea llevada cabo por el medio.

En tercer lugar, es significativo recalcar que en otros contextos internacionales se han explotado otros sistemas aún no detectados en el caso español, como:

- vídeos que explican las decisiones editoriales, Repubblica domani en el medio La repubblica http://video.repubblica.it/rubriche/repubblica-domani

- listas abiertas de temas en producción, Open news list en The guardian

http://www.theguardian.com/news/series/open-newslist

- retransmisión online de los consejos de redacción en The Baltimore Sun

http://www.baltimoresun.com/bal-livestream-videohtmlstory.html

- contribución de los ciudadanos a los consejos de redacción mediante las posibilidades que brinda la Red, Rue89 http://www.rue89.com/participez-a-la-conference-deredaction-en-ligne

Los medios españoles deben experimentar con otras fórmulas como los vídeos editoriales, las listas abiertas de temas o la retransmisión online de los consejos de redacción

No hay duda de que los condicionantes económicos y de personal juegan un papel clave en la viabilidad de la introducción de nuevos mecanismos, pero los medios españoles deberían valorar la posibilidad de experimentar con estas fórmulas y otras que emerjan en el futuro. Por último, se señala la conveniencia de potenciar la visibilidad y la información sobre la existencia de estos sistemas con el fin que los lectores sean plenamente conscientes de su utilidad para monitorizar la calidad del trabajo periodístico.

Trabajos futuros deberán examinar de forma sistemática y longitudinal la presencia y el uso de las iniciativas emergentes de rendición de cuentas en otros contextos periodísticos, con la intención de llevar a cabo estudios comparativos transnacionales. También se plantea la pertinencia de triangular (Hesse-Biber, 2010) la metodología empleada con entrevistas en profundidad (King; Horrocks, 2010) con expertos, profesionales y lectores, con el objetivo de conocer sus percepciones sobre la creación, diseño e impacto de estos nuevos formatos que fomentan la autorregulación, transparencia y participación del público.

\section{Notas}

1. Detalles sobre el caso:

http://goo.gl/tuYwZZ

Mensaje de Twitter del director de El periódico de Catalunya, Enric Hernàndez, a sus seguidores:

https://goo.gl/ZuJees

2. Cuentas de Eldiario.es correspondientes al primer año (2013) y el segundo año (2014) de actividad:

http://www.eldiario.es/escolar/gastamos-dinero-anoeldiarioes_6_176442363.html

http://www.eldiario.es/escolar/cuentas-eldiarioessegundo-aniversario_6_303829638.html

Se debe potenciar la visibilidad de estos sistemas para que los usuarios sean conscientes de su utilidad para monitorizar la calidad del trabajo periodístico

\section{Bibliografía}

Alsius, Salvador (1999). Codis ètics del periodisme televisiu. Barcelona: Pòrtic. ISBN: 9788473065702

Alsius, Salvador; Mauri-Ríos, Marcel; Rodríguez-Martínez, Ruth (2011). "Spain: A diverse and asymmetric landscape". En: Eberwein, Tobias; Fengler, Susanne; Lauk, Epp; LeppikBork, Tanja (eds.). Mapping media accountability - in Europe and beyond. Colonia: Helbert Von Halem Verlag, pp. 155-167. ISBN: 9783869620381

Alsius, Salvador; Salgado, Francesc (eds.) (2010). La ética informativa vista por los ciudadanos. Contraste de opiniones entre los periodistas y el público. Barcelona: UOC. ISBN: 9788497889353

Altheide, David L. (1996). Qualitative media analysis. Thousand Oaks, California: Sage. ISBN: 9780761901990

Aznar, Hugo (1999). Ética y periodismo: códigos, estatutos y otros documentos de autorregulación. Barcelona: Paidós. ISBN: 8449306531

Barroso, Porfirio (1984). Códigos deontológicos de los medios de comunicación (prensa, radio, televisión, cine, publicidad y relaciones públicas). Madrid: Ediciones Paulinas Verbo Divino. ISBN: 8428509719

Bertrand, Claude-Jean (2000). Media ethics and accountability systems. Londres: New Brunswick. ISBN: 9781560004202

Bichler, Klaus; Harro-Loit, Halliki; Karmasin, Matthias; Kraus, Daniela; Lauk, Epp; Loit, Urmas; Fengler, Susanne; Schneider-Mombaur, Laura (2012). Best practice guidebook: media accountability and transparency across Europe. http://www.mediaact.eu/fileadmin/user_upload/ Guidebook/guidebook.pdf

Bryman, Alan (2012). Social research methods (4a ed.). Oxford: Oxford University Press. ISBN: 9780199588053

Christians, Clifford G.; Glasser, Theodore L.; McQuail, Denis; Nordenstreng, Kaarle; White, Robert A. (2009). Normative 
theories of the media. Journalism in democratic societies. Urbana: University of Illinois Press. ISBN: 9780252076183

Domingo, David; Heinonen, Ari (2008). "Weblogs and journalism. A typology to explore the blurring boundaries". Nordicom review, v. 29, n. 1, pp. 3-15.

http://jclass.umd.edu/classes/jour698m/domingoblogs.pdf

Eberwein, Tobias (2010). "Von Holzhausen nach Blogville - und zurück medienbeobachtung in tagespresse und weblogs". En: Eberwein, Tobias; Müller, Daniel (eds.). Journalismus und öffentlichkeit. Wiesbaden: Verlag, pp. 143-165. ISBN: 9783531157597

Evers, Hubb; Groenhart, Harmen; Van-Groesen, Jan (2010). The news ombudsman. Watch-dog or decoy? Diemen: AMB. ISBN: 9789079700202

http://newsombudsmen.org/blog/the-news-ombudsmanwatchdog-or-decoy

Fengler, Susanne; Eberwein, Tobias; Mazzoleni, Gianpietro; Porlezza, Colin; Russ-Mohl, Stephan (2014). Journalists and media accountability. An international study of news people in the digital age. Nueva York: Peter Lang Publishing. ISBN: 9781433122811

García-Avilés, José-Alberto (1996). Periodismo de calidad. Estándares informativos en la CBS, NBC y $A B C$. Barañain, Navarra: Eunsa. ISBN: 9788431314446

Goodman, Leo A. (1961). "Snowball sampling". Annals of mathematical statistics, v. 32, n. 1, pp. 148-170.

http://dx.doi.org/10.1214/aoms/1177705148

Herrera-Damas, Susana (2013). “Indicaciones recurrentes en las normativas para el uso periodístico de las redes sociales". El profesional de la información, v. 22, n. 1, pp. 46-53. http://dx.doi.org/10.3145/epi.2013.ene.06
Hesse-Biber, Sharlene-Nagy (2010). Mixed methods research: merging theory with practice. Nueva York: The Guilford Press. ISBN: 9781606232590

King, Nigel; Horrocks, Christine (2010). Interviews in qualitative research. Thousand Oaks, California: Sage. ISBN: 978 1412912570

Maciá-Barber, Carlos (2006). "Un modelo de defensor del lector, del oyente y del telespectador para el perfeccionamiento del ejercicio del periodismo en España". Comunicación y sociedad, v. 19, n. 1, pp. 47-66.

http://www.unav.es/fcom/communication-society/es/ articulo.php?artid $=56$

Nordenstreng, Kaarle; Hannikainen, Lauri (1984). The mass media declaration of Unesco. Norwood, New Jersey: Ablex. ISBN: 9780893910778

Pastor, Lluís; Martínez-Martínez, Silvia (2013). “La retórica al servicio del usuario: estudio de los comentarios de los lectores en Elpais.com, Abc.es y Lavanguardia.es". El profesional de la información, v. 22, n. 2, pp. 113-121.

http://dx.doi.org/10.3145/epi.2013.mar.03

Pérez-Fuentes, Juan-Carlos (2004). Ética periodística. Principios, códigos deontológicos y normas complementarias. Leioa: Servicio editorial de la Universidad del País Vasco. ISBN: 9788483735947

Starck, Kenneth (2010). "The news ombudsman: viable or vanishing?". En: Eberwein, Tobias; Müller, Daniel (Eds.). Journalismus und öffentlichkeit. Wiesbaden: Verlag, pp. 109-118. ISBN: 9783531157597

Wimmer, Roger D.; Dominick, Joseph R. (2000). Mass media research: an introduction (6a ed.). Nueva York: Wadsworth Publishing Company. ISBN: 9780534560072

\section{Nueva colección EPI Scholar}

\section{Libros académicos y científicos de Información y Documentación}

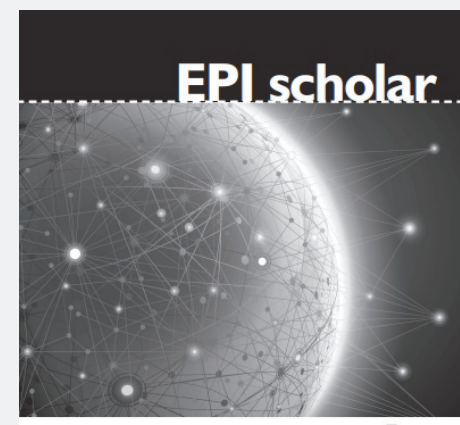

CIBERMETRÍA MIDIENDO EL ESPACIO RED ENRIQUE ORDUNA-MALEA ISIDRO F. AGUILLO

\author{
Primer título: \\ Cibermetría. Midiendo el espacio red \\ de Enrique Orduña-Malea e Isidro F. Aguillo
}

A pesar del crecimiento de la disciplina de la Cibermetría desde mediados de la década de los noventa, son escasos los libros académicos o manuales dedicados en exclusiva a la misma desde un contexto de las ciencias de la información y documentación. Este libro pretende cubrir este claro hueco en la literatura tanto nacional como internacional. 